\title{
High-resolution imaging of biotite using focal series exit wavefunction restoration and the graphene mechanical exfoliation method
}

\author{
W. Bower ${ }^{1}$, W. $\operatorname{Head}^{1}$, G. T. R. Droop ${ }^{1}$, R. Zan ${ }^{2, \dagger}$, R. A. D. Pattrick ${ }^{1}$, P. Wincott ${ }^{1}$ And S. J. Haigh $^{2, *}$ \\ 1 School of Earth, Atmospheric and Environmental Sciences and the Williamson Research Centre, The University \\ of Manchester, Manchester M13 9PL, UK \\ 2 School of Materials, The University of Manchester, Manchester M13 9PL, UK
}

[Received 5 November 2013; Accepted 16 March 2015; Associate Editor: P. O. Brien]

\section{ABSTRACT}

We have applied mechanical exfoliation for the preparation of ultra-thin samples of the phyllosilicate mineral biotite. We demonstrate that the 'scotch tape' approach, which was made famous as an early method for production of single-atom-thick graphene, can be used for production of sheet-silicate specimens that are sufficiently thin to allow high-resolution transmission electron microscope (HRTEM) imaging to be achieved successfully while also being free from the specimen preparation artefacts that are often caused by ion-beam milling techniques. Exfoliation of the biotite parallel to the (001) planes has produced layers as thin as two structural TOT units thick $(\sim 2 \mathrm{~nm})$. The minimal specimen thickness enabled not only HRTEM imaging but also the application of subsequent exit wavefunction restoration to reveal the pristine biotite lattice. Exit wavefunction restoration recovers the full complex electron wave from a focal series of HRTEM images, removing the effects of coherent lens aberrations. This combination of methods therefore produces images in which the observed features are readily interpreted to obtain atomic resolution structural information.

KEYWORDs: biotite, exfoliation, focal series, high-resolution transmission electron microscopy, graphene.

\section{Introduction}

RECENT improvements in the resolution of transmission electron microscopes (TEMs) have demonstrated a need for increasingly thin highquality samples, with thicknesses $<\sim 10 \mathrm{~nm}$ often being preferred (Urban, 2008). Specimen thickness is particularly important when operating the TEM at a lowered accelerating voltage to reduce beam-induced knock-on damage or when performing electron energy loss spectroscopy (EELS), but reaching the required thickness

\footnotetext{
* E-mail: sarah.haigh@manchester.ac.uk

$\dagger$ Present address: Department of Physics, Faculty of Arts and Sciences, Niğde University, 51000 Niğde, Turkey

DOI: 10.1180/minmag.2015.079.2.11
}

while retaining the perfect crystal structure is very challenging. Thinning of a material in preparation for HRTEM is often achieved by bombardment with high-energy argon ions. However, this can lead to significant lattice damage and surface amorphization, even on robust phases such as silicates (McCaffrey et al., 2001), and may lead to the development of artefacts as well as difficulties in the identification of original lattice deformation features or defects. Phyllosilcates, such as micas and clays are built of sheets of oxygen-bonded tetrahedral silica and octahedral metal layers (TOT), weakly held together by interlayers containing high coordination cations, such as $\mathrm{K}$, water or electrostatic charges. Biotite is a $2: 1$ phyllosilicate which, like many sheet silicate minerals, has a strong tendency for basal-plane cleavage. The 


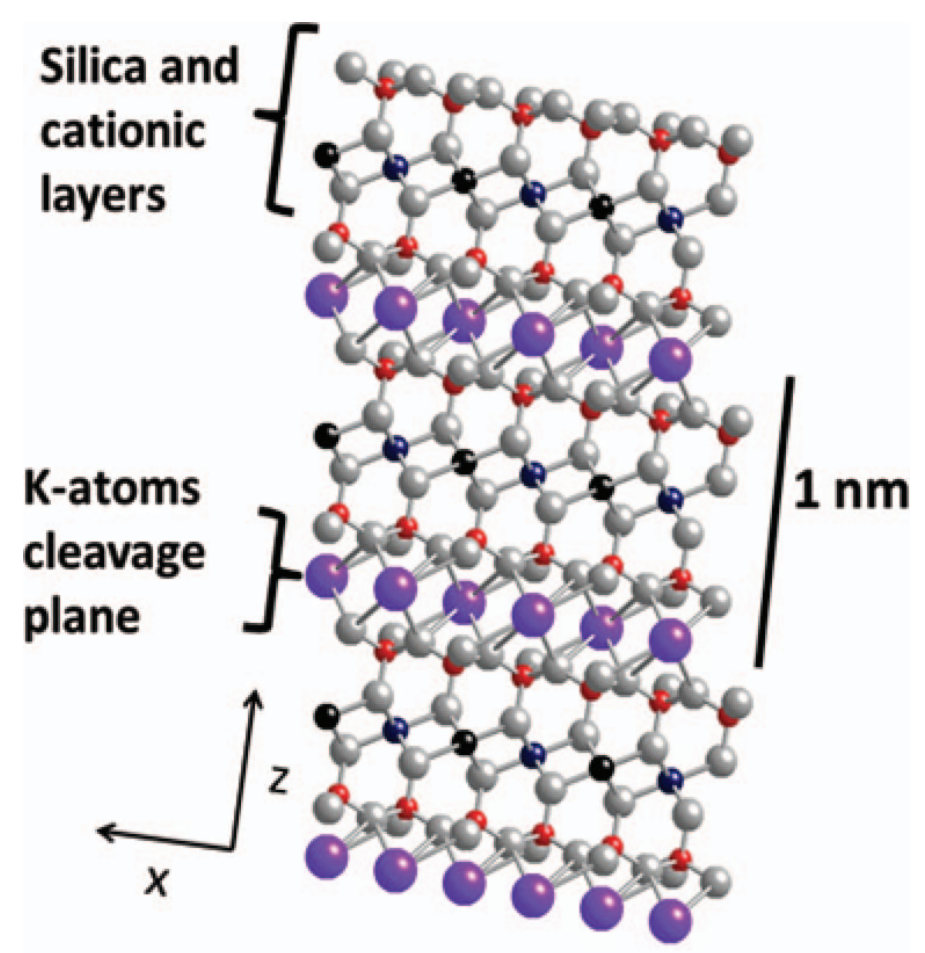

FIG. 1. The 2:1 phyllosilicate biotite structure (viewed parallel to [010]) showing the octahedral Fe+Mg (black) and tetrahedral $\mathrm{Si}+\mathrm{Al}$ (red) layers joined by high-coordination $\mathrm{K}$ atoms (purple). Oxygen atoms are grey. Three complete (TOT) structural layers are shown, each having a thickness of $\sim 1 \mathrm{~nm}$ in the [001] direction.

structure of biotite comprises TOT layers containing mainly $\mathrm{Mg}$, $\mathrm{Ti}$ and $\mathrm{Fe}$, bonded by interstitial $\mathrm{K}$ atoms (Fig. 1). This structure allows the easy cleavage of the structure parallel to these TOT layers. This weak interplanar bonding has been exploited in this work to produce ultra-thin crystal flakes via mechanical exfoliation. This cleavage approach is also referred to as the 'Scotch Tape' method and has attracted considerable attention from its successful use for the preparation of single-layer graphene (Novoselov et al., 2004) and more recently for other twodimensional inorganic crystals (Geim and Grigorieva, 2013).

In this study we demonstrate the use of the mechanical exfoliation method for the preparation of phyllosilicate samples suitable for HRTEM lattice imaging. The method has the considerable advantage of avoiding damage induced by other specimen-preparation techniques such as argon ion-beam polishing or focussed ion beam (FIB) milling. This absence of sample preparation artefacts is particularly important when studying point defects or structural degradation produced as a result of radiation damage, extreme environmental conditions or chemical attack (Ma et al., 1998).

\section{Biotite characterization}

The mineral we have used to demonstrate this technique is biotite, taken from a $2 \mathrm{Ba}$ almandinegrunerite-biotite sample from near Tunaberg, Sweden. The sample was chosen because the host rock was well characterized (Pattrick et al. 2013) and consisted of large single crystals $(\sim 2 \mathrm{~cm}$ in diameter). X-ray diffraction (XRD) analysis using a Bruker D8 Advance diffractometer confirmed the mineral as biotite mica (Cruciani and Zanazzi, 1994; Brigatti et al., 2008). The bulk chemistry of the biotite was established by means of electron probe microanalysis (EPMA) (Reed, 2010), using a Cameca SX100 microsonde with a beam current of $20 \mathrm{nA}$ and an acceleration voltage of $15 \mathrm{keV}$. Standards used for biotite were fayalite $(\mathrm{Fe})$, tephroite $(\mathrm{Mn})$, wollastonite $(\mathrm{Ca}$ and $\mathrm{Si})$, rutile (Ti), K-feldspar $(\mathrm{K})$, cordierite $(\mathrm{Al})$, jadeite $(\mathrm{Na})$, periclase $(\mathrm{Mg})$ and rutile $(\mathrm{Ti})$. The average of 
40 point analyses showed the composition of the biotite to be: $\mathrm{K}_{0.94} \mathrm{Na}_{0.03} \mathrm{Fe}_{1.95} \mathrm{Mg}_{0.91} \mathrm{Mn}_{0.05} \mathrm{Ti}_{0.02}$ $\mathrm{Al}_{1.25} \mathrm{Si}_{2.84} \mathrm{O}_{10}(\mathrm{OH})_{2}$ and this composition falls close to the annite-phlogopite binary join (see Fleet, 2003). Flakes of mica $\sim 20-30 \mu \mathrm{m}$ thick, were extracted from the rock for this study.

\section{Exfoliation of biotite}

In this work we report use of the mechanical exfoliation sample preparation technique, as described for graphene in Novoselov et al., (2004) to produce ultra-thin layers of biotite. In this method, a high tack/low stain adhesive tape is pressed and peeled onto itself repeatedly so as to pull off increasingly thinner layers of material. The tape with the thinnest layers of material is then stuck down onto an oxidized silicon substrate. Following this, the tape is then dissolved in methyl isobutyl ketone heated to $80^{\circ} \mathrm{C}$, leaving the exfoliated flakes on the silica. A final tape peel is employed to remove the thickest flakes from the substrate although the remaining material contains biotite flakes with a range of thicknesses.

Optical imaging was used to identify which regions of the exfoliated material were ultra-thin from their characteristic violet-blue colour (Fig. 2a). Very thin flakes are often highly transparent so have poor contrast in the optical microscope. However, the correct choice of optical wavelength and the added path of the $\mathrm{SiO}_{2}$ substrate shifts the optical interference pattern, allowing even monolayer graphene to be clearly visible (Blake et al., 2007); a similar effect was expected and found for biotite. A wavelength of $90 \mathrm{~nm}$ and crossed polars was chosen so as to give optimal contrast for the thinnest flakes when supported on a silica wafer with a $300 \mathrm{~nm}$ surface oxide.

\section{Atomic force microscopy analysis of ultra- thin biotite}

An atomic force microscope (AFM) (Binning et al., 1986) was used to measure the height of the exfoliated biotite films relative to the silica substrate (see Fig. 2). The machine used was a Digital Instruments Multimode IIIA operated in tapping mode. The tips were Veeco RTESPA10 which are $\mathrm{p}(\mathrm{n})$ doped Si $1-10 \mathrm{ohm} \cdot \mathrm{cm}$ with a nominal frequency of $280 \mathrm{kHz}$, a nominal force constant of $40 \mathrm{~N} / \mathrm{m}$, a mean tip radius of $10 \mathrm{~nm}$ and $\mathrm{Al}$ reflective back coating. Transverse AFM scans across the surface of two of the thinnest flakes revealed average step heights of $2.180 \pm 0.2$ and $1.826 \pm 0.2 \mathrm{~nm}$ respectively. These values are close to the thickness of two complete $\sim 1 \mathrm{~nm}$ biotite TOT layers. The sharp peak visible in Fig. $2 c$ is considered to be a region where the biotite has folded or creased. When other thicker flakes of the sample were measured, thicknesses increased in units of $\sim 2 \mathrm{~nm}$ in height, with no evidence of fragments $1,3,5$ or $7 \mathrm{~nm}$ thick. We suggest that surface charges may be the reason for the observation of flakes with even numbers of TOT layers (whole unit cells).

\section{Flake transfer}

Transfer of the flakes from the silica substrate onto a support grid suitable for transmission electron microscope (TEM) imaging was achieved by the method described by Meyer et al. (2008) as summarized in Fig. 3. The exfoliated sample was coated in a thin poly(methyl methacrylate) (PMMA) protective film, then heated to $110^{\circ} \mathrm{C}$ for a few minutes to improve adhesion. After checking the position of the region of interest in an optical microscope, a circle was scribed into the PMMA around the biotite flakes chosen for transfer. $\mathrm{KOH}$ was then used to chemically etch away the underlying $\mathrm{SiO}_{2}$ layer so that the biotite-PMMA composite film could be floated free from the Si wafer in distilled water. After rinsing in distilled water the sample was transferred to a quantifoil TEM carbon support grid. The PMMA support layer was then removed by dissolving in acetone. Finally the sample was dried using a Critical Point Dryer (CPD), with acetone as an intermediate agent, before being replaced with liquid $\mathrm{CO}_{2}$ and heated to $40^{\circ} \mathrm{C}$. The successful transfer of a flake only a few TOT layers thick, identified using optical microscope imaging, onto a support grid to enable high-resolution TEM imaging is illustrated in Fig. 3. It is difficult to distinguish the ultra-thin flake region optically after transfer (Fig. $3 c-e$ ), but its location on the grid can be deduced from the position of the thicker flakes, as the relative locations of the flakes are largely preserved after transfer. The thin biotite region is easily identified at low magnification in the TEM using a small objective aperture to increase contrast (Fig. $3 f$ ).

\section{High-resolution imaging}

The ultrathin biotite flakes were analysed at high resolution using the Titan ${ }^{\circledR}$ G2 80-200 (S) TEM 

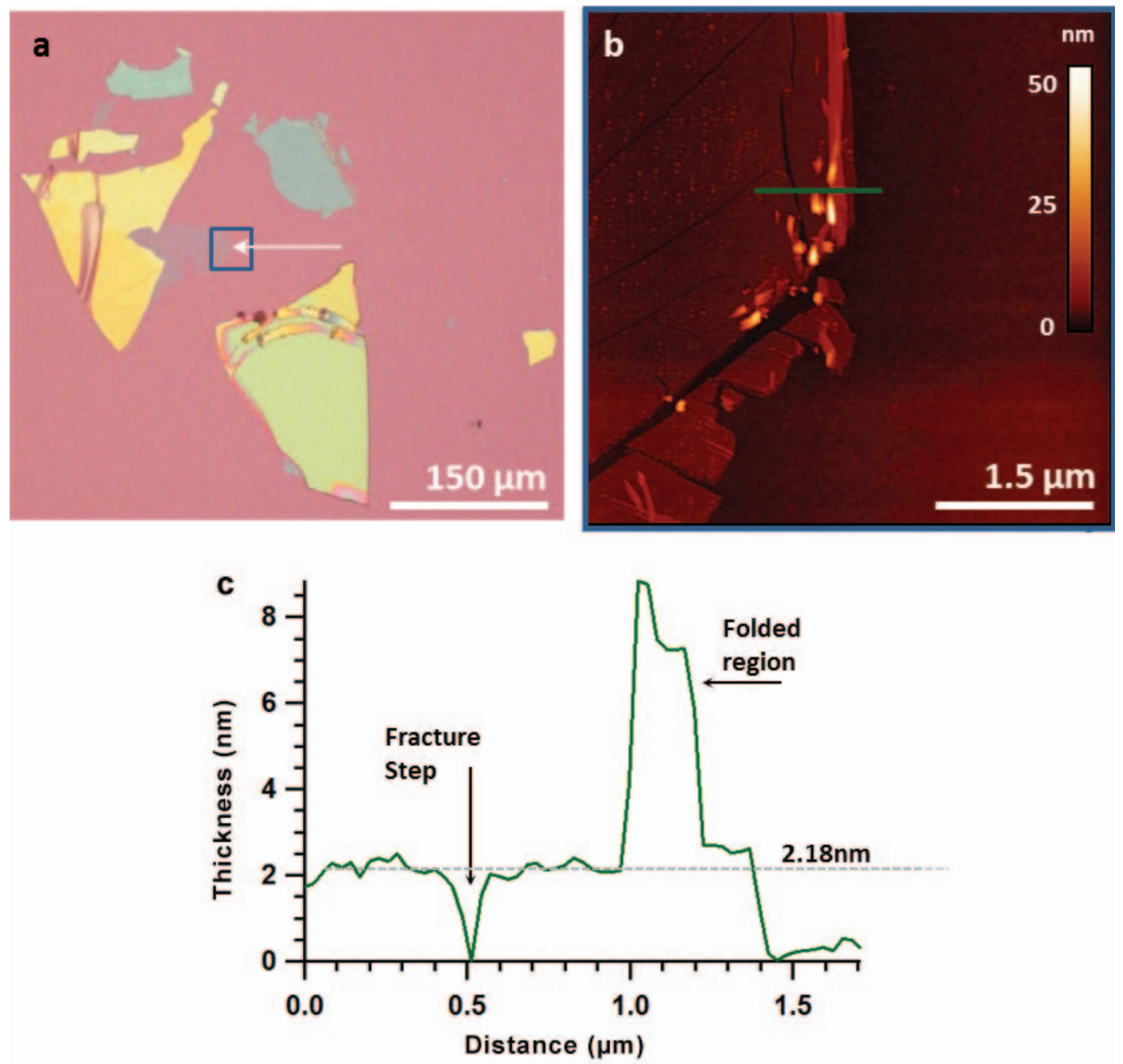

FIG. 2. Atomic force microscopy analysis of the exfoliated flakes. (a) Optical microscope image showing exfoliated biotite flakes on a silica substrate. The thinnest flake highlighted by the white arrow has a characteristic violet-blue interference colour while multilayer flakes have blue, orange or pale green colours as the sample thickness increases. The blue square indicates the region of flake edge analysed by AFM in $(b)$ and $(c)$. (b) AFM surface image in tapping mode, detailing the edge of the ultrathin flake. The green line marks the position of the transect shown in $(c)$, passing over the folded region and beyond the edge of the sample. (c) AFM line scan across the edge of the flake; note the folded region accounting for a thickness of $>8 \mathrm{~nm}$. The average thickness of the exfoliated biotite in the unfolded region is $2.18 \pm 0.2 \mathrm{~nm}$, indicative of 2 TOT structural layers (Fig. 1).

in the School of Materials, University of Manchester. The instrument was operated in the conventional TEM imaging mode using an accelerating voltage of $200 \mathrm{kV}$. Imaging conditions were chosen to obtain a reasonable signalto-noise ratio while limiting beam-induced radiation damage (specimen pixel size of $0.0216 \mathrm{~nm}$, beam current of $3.5 \mathrm{nA}$, exposure of $1 \mathrm{~s}$ and an imaging dose of $\sim 100$ electrons $\AA^{-2}$ ). Using these imaging parameters it was possible to acquire $>30$ images without seeing any electron beam-induced sample damage although significant damage was observed after 100 images (Fig. 4). The images here were all acquired for biotite viewed along [001] but several other zone axes are accessible by tilting the sample using a standard double tilt 

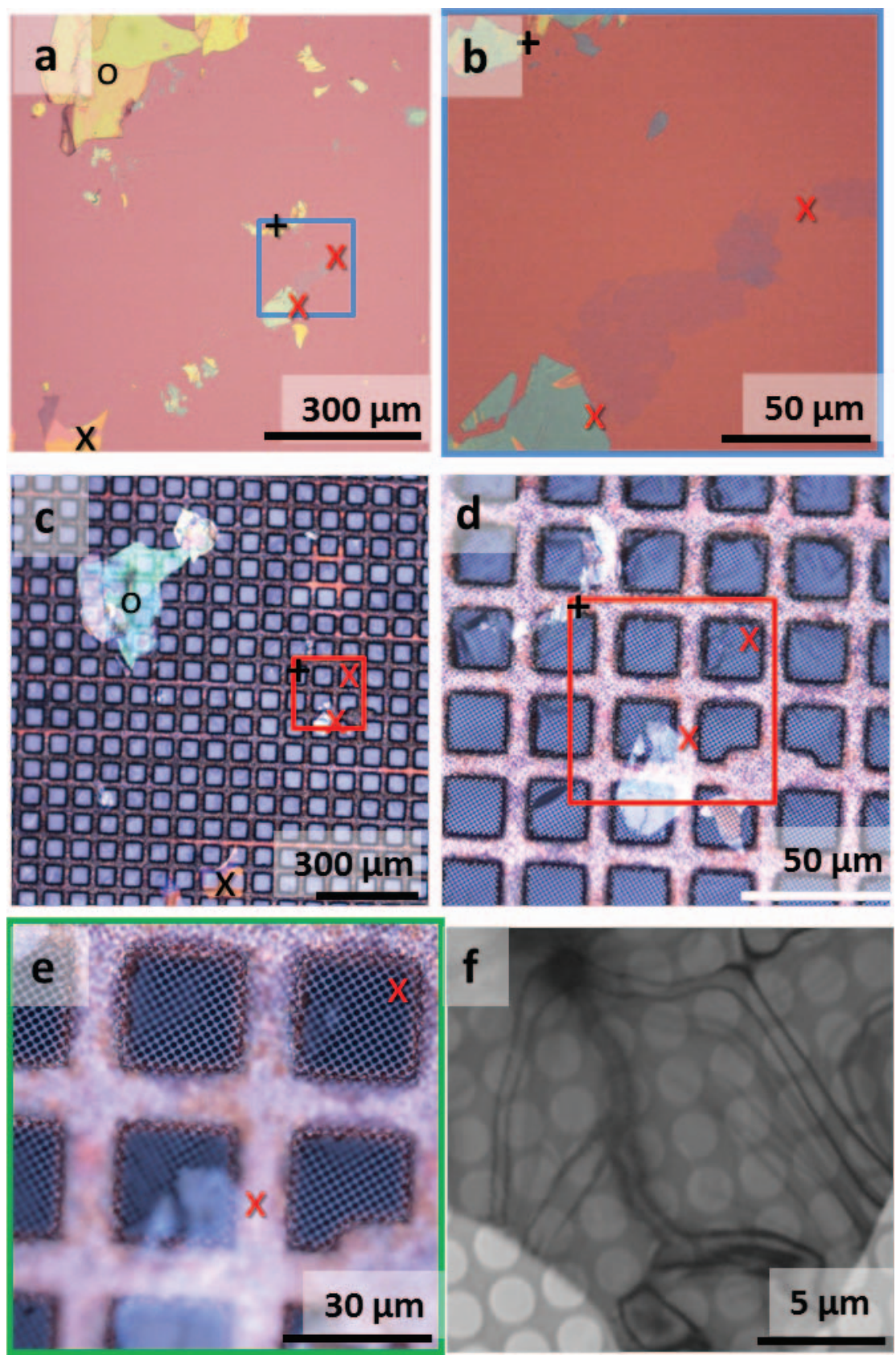

Fig. 3. Illustrating the process of flake transfer from the silica substrate to the TEM support grid. Optical microscope images showing exfoliated biotite flakes on a silica substrate $(a$ and $b)$ and after transfer to the TEM support grid $(c-e)$. The thinnest flakes are positioned between the red crosses and have the characteristic violet-blue colour. The black symbols $(\mathrm{o},+$ and $\mathrm{x})$ highlight positions on thicker flakes, demonstrating that relative positions are largely preserved before and after transfer. The blue square in $(a)$ indicates the region-enlarged edge $(b)$. The red square in $(c)$ and $(d)$ indicates the region-enlarged edge $(e)$. $(f)$ Low-magnification TEM image showing the regular array of round holes in the quantifoil TEM support grid and a thin biotite flake. 

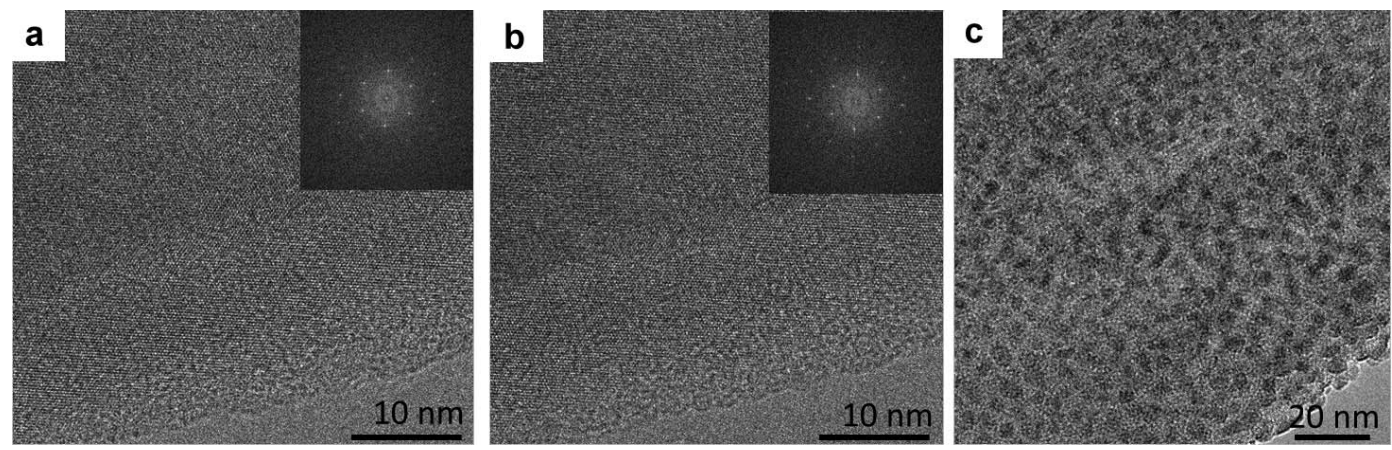

FIG. 4. High-resolution TEM images demonstrating the absence of electron beam-induced radiation damage during focal series acquisition. Images were acquired at nominally the same defocus $(a)$ before and $(b)$ after the acquisition of the 20 image focal series data used to restore the exit wavefunction shown in Fig. 5(a). The Fourier transforms of the images (inset in $a$ and $b$ ) show there was no loss of lattice information during image series acquisition. (c) The same area after $>100$ images: the mottled appearance of the image indicates that the sample is damaged severely.

TEM specimen holder or by taking advantage of folds and creases in the sheets.

High-resolution TEM images often suffer from the effects of high spatial frequency oscillations in the contrast transfer function due to coherent aberrations of the objective lens. When the sample is sufficiently thin, exit wavefunction restoration from a series of TEM images allows these effects to be compensated providing local structural information in the phase of the exit wavefunction (Coene et al., 1992, 1996). Focal series data sets consisting of 10-20 high-resolution transmission electron microscope images with a uniform focal step $(\sim 10 \mathrm{~nm})$ were recorded close to the Scherzer defocus $(-39 \mathrm{~nm}$ for $200 \mathrm{kV}$ electrons and a spherical aberration of $0.6 \mathrm{~mm}$ ). Highresolution lattice images acquired at the same defocus value before and after a 20 image focal series are compared in Fig. $4 a$ and $b$. No beaminduced damage is visible as a result of focal series data acquisition and the inset Fourier Transforms (FTs) for the images appear identical, demonstrating no loss of information transfer. However, after the acquisition of $\sim 100$ images, damage to the crystal was clearly visible (Fig. 4c). Exit wavefunction restorations were calculated from the focal series data sets using a Wiener filter approach (Saxton, 1988) implemented using the FTSR commercial software (www.hremresearch.com) within the Gatan Digital Micrograph program. Prior to exit wavefunction restoration the images were aligned using a phase correlation function/ phase contrast index function to correct for specimen drift and to determine the precise focal step variations between images (Meyer et al., 2002,
2004). The modulation transfer function for the CCD camera was determined independently and compensated as part of the restoration process (Meyer et al. 2000). The phase of the exit wavefunction restored using the focal series data set acquired between the images in Fig. $4 a$ and $b$ is shown in Fig. 5a. An advantage of restoring the full complex exit wavefunction is that the uncertainty of comparison to image simulations is reduced. Image simulations were performed using the multislice method (Goodman and Moodie, 1974; Lynch and O'Keefe, 1972) for the phlogopite crystal structure (Takeda and Ross, 1975). These confirmed that the images and restored exit wavefunction had the contrast expected from the phyllosilicate structure with a specimen thickness of $<20 \mathrm{~nm}$ (inset Fig. 5b). It is interesting to note that restoration of the exit wavefunction has the additional advantage of improving the signal-to-noise ratio in the images and that this enables the weaker $\{110\}$ and $\{020\}$ reflections to appear in the FT of the restored exit wavefunction (Fig. 5c) even though they are not visible above the noise in the FT of an individual HRTEM image (inset Figs $5 a, b$ ). The diffraction pattern from the same sample region has a similar contrast to the FT of the restored exit wave (Fig. $5 c, d$ ) with $\{110\}$ and $\{020\}$ reflections having considerably weaker intensity than $\{200\}$ (consistent with dynamical diffraction effects for sample thicknesses of $<20 \mathrm{~nm}$ ).

\section{Summary}

Our results demonstrate that mechanical exfoliation combined with identification of the thinnest 


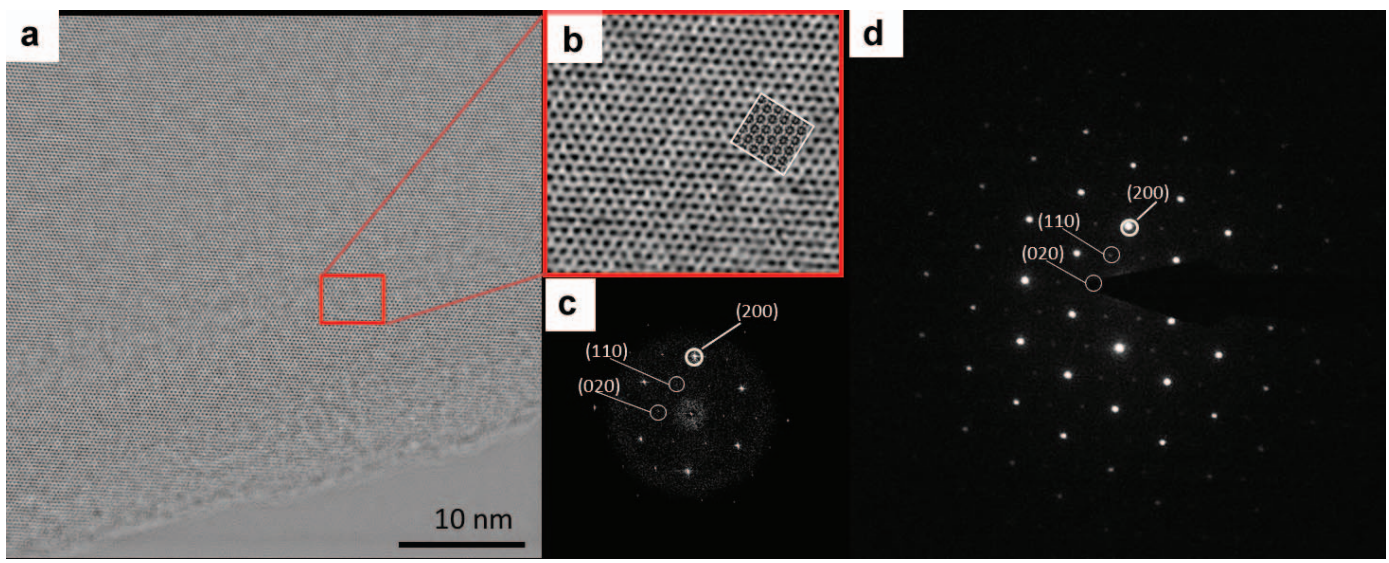

FIG. 5. (a) Phase of the exit wavefunction restored from a focal series of 20 high-resolution TEM images for biotite viewed along [001]. (b) An enlargement of the region shown by the red rectangle in (a) with the simulated phase of the exit wavefunction shown overlaid. (c) Fourier transform of the exit wavefunction revealing the presence of the weaker $\{110\}$ and $\{020\}$ lattice reflections in the restored exit wave which were not visible in the Fourier transform of a single high-resolution image due to the poorer signal-to-noise ratio (inset Fig. 4a). (d) Electron diffraction pattern from the same flake illustrating the presence of both the weaker reflections $(\{110\},\{020\})$ and the stronger reflections such as $\{200\}$.

flakes using optical imaging and transfer to standard TEM support films can provide specimens suitable for high-quality lattice imaging in phyllosilicates. This sample-preparation approach avoids the need for argon-ion polishing and hence the possibility of artefacts introduced as a result of ion-beam milling. The samples were found to be sufficiently stable to allow the routine use of highresolution focal series data acquisition and exit wavefunction restoration. This novel approach is suitable for any sheet silicates with weak interlayer bonding. The properties of clay minerals are very dependent on structure and there is considerable interest in the use of clay in retaining radionuclides in a Geological Disposal Facility. Radiation damage investigations involving synchrotron radiation have implied that there are major effects on the "metal layers, including breakdown of $\mathrm{OH}$ groups, Fe-redox changes, defect generation and amorphisation" (Ewing et al., 2000; Pattrick et al., 2013). In using the TEM to assess structural damage to the clays induced by deliberate radiation exposure, it is essential to eliminate the possibility that the observed changes are sample-preparation artefacts. This necessitates the production of thin, defect-free crystals and has motivated this work but we recognize that the ability to produce very thin, high-quality TEM samples from sheet silicates has much wider applications.

\section{Acknowledgements}

The authors dedicate this paper to Paul Wincott, a much loved colleague. William Head is supported by the Engineering and Physical Sciences Research Council (EPSRC) Nuclear FiRST Doctoral Training Centre and William Bower by a NERC PhD Studentship. Dr Haigh thanks EPSRC and the Defence Threat Reduction Agency (US) for funding support. The authors are grateful to Edward Lewis for his technical advice during sample manipulation and Alan Harvey for TEM support.

\section{References}

Binning, G., Quate, C.F. and Gerber, C. (1986) Atomic force microscope. Physical Review Letters, 56, 930-933.

Blake, P., Hill, E.W., Castro Neto, A.H., Novoselov, K.S. and Jiang, D. (2007) Making graphene visible. Applied Physics Letters, 91, 063124.

Brigatti, M.F., Guidotti, C.V., Malferrari, D. and Sassi, F.P. (2008) Single-crystal X-ray studies of trioctahedral micas coexisting with dioctahedral micas in metamorphic sequences from western Maine. American Mineralogist, 93, 396-408.

Coene, W., Janssen, G., Op de Beeck, M. and Van Dyck, D. (1992) Phase retrieval through focus variation for ultra-resolution in field-emission transmission 
electron-microscopy. Physical Review Letters, 69, 3743-3746.

Coene, W.M.J., Thust, A., Op de Beeck, M. and Van Dyck, D. (1996) Maximum-likelihood method for focus-variation image reconstruction in high resolution transmission electron microscopy. Ultramicroscopy, 64, 109-135.

Cruciani, G. and Zanazzi, P.F. (1994) Cation partitioning and substitution mechanisms in 1M-phlogopite: a crystal chemical study. American Mineralogist, 78, 289-301.

Goodman, P. and Moodie, A.F. (1974) Numerical evaluation of N-Beam wave function in electron scattering by the multislice method. Acta Crystallograhica, A30, 280-290.

Ewing, R.C., Meldrum, A., Wang, L.M. and Wang, S.X. (2000) Radiation-induced amorphization. Transformation Processes in Minerals, 39, 319-361.

Fleet, M.E. (2003) Rock-Forming Minerals, Volume $3 A$ Sheet Silicates: Micas. The Geological Society, London, $758 \mathrm{pp}$.

Geim, A.K. and Grigorieva, I.V. (2013) Van der Waals heterostructures. Nature, 499, 419-425.

Lynch, D.F. and O'Keefe, M.A. (1972) N-Beam lattice images. 2. Methods of calculation. Acta Crystallographica, A28, 536-548.

Ma, C. Fitzgerald, J.D., Eggleton, R.A. and Llewellyn, D.J. (1998) Analytical electron microscopy in clays and other phyllosilicates: Loss of elements from a 90-nm stationary beam of 300-keV electrons. Clays and Clay Minerals, 46, 301-316.

McCaffrey, J.P., Phaneuf, M.W. and Madsen, L.D. (2001) Surface damage formation during ion-beam thinning of samples for transmission electron microscopy. Ultramicroscopy, 87, 97-104.

Meyer, J.C., Girit, C.O., Crommie, M.F. and Zettl, A. (2008) Imaging and dynamics of light atoms and molecules on graphene. Nature, 454, 319-322.

Meyer, R.R., Kirkland, A.I., Dunin-Borkowski, R.E. and Hutchison, J.L. (2000) Experimental characterisation of CCD cameras for HREM at $300 \mathrm{kV}$. Ultramicroscopy, 85, 9-13.

Meyer, R.R., Kirkland, A.I. and Saxton, W.O. (2002) A new method for the determination of the wave aberration function for high resolution TEM 1. Measurement of the symmetric aberrations. Ultramicroscopy, 92, 89-109.

Meyer, R.R., Kirkland, A.I. and Saxton, W.O. (2004) A new method for the determination of the wave aberration function for high-resolution TEM. 2. Measurement of the antisymmetric aberrations. Ultramicroscopy, 99, 115-123.

Novoselov, K.S., Geim, A.K., Morozov, S.V., Jiang, D., Zhang, Y., Dubonos, S.V., Grigorieva, I. V. and Firsov, A.A. (2004) Electric field effect in atomically thin carbon films. Science, 306, 666-669.

Pattrick, R.A.D., Charnock, J.M., Geraki, T., Mosselmans, J.F.W., Pearce, C.I., Pimblott, S. and Droop, G.T.R. (2013) Alpha particle damage in biotite characterized by microfocus X-ray diffraction and $\mathrm{Fe} \mathrm{K}$-edge X-ray absorption spectroscopy. Mineralogical Magazine, 77, 2867-2882.

Reed, S.J.B. (2010) Electron Microprobe Analysis and Scanning Electron Microscopy in Geology. Cambridge University Press, Cambridge, UK.

Saxton, W.O. (1988) Accurate atom positions from focal and tilted beam series of high-resolution electronmicrographs. Scanning Microscopy, 2, 213-224.

Takeda, H. and Ross, M. (1975) Mica polytypism: Dissimilarities in the crystal structures of coexisting $1 M$ and $2 M 1$ biotite. American Mineralogist, 60, 1030-1040.

Urban, K.W. (2008) Studying atomic structures by aberration-corrected transmission electron microscopy. Science, 321(5888), 506-510. 